\title{
Corela
}

Cognition, représentation, langage

9-1 | 2011

Vol. $9, n^{\circ} 1$

\section{Test : La sous-spécification flexionnelle des bases de dérivation en question à la lumière de la morphologie bretonne}

Pierre-Yves Kersulec

\section{(2) OpenEdition}

Journals

Édition électronique

URL : https://journals.openedition.org/corela/1980

DOI : $10.4000 /$ corela. 1980

ISSN : 1638-573X

Éditeur

Cercle linguistique du Centre et de l'Ouest - CerLICO

Référence électronique

Pierre-Yves Kersulec, «Test : La sous-spécification flexionnelle des bases de dérivation en question à la lumière de la morphologie bretonne », Corela [En ligne], 9-1 | 2011, mis en ligne le 06 juin 2011, consulté le 24 juin 2021. URL : http://journals.openedition.org/corela/1980 ; DOI : https://doi.org/ $10.4000 /$ corela.1980

Ce document a été généré automatiquement le 24 juin 2021.

\section{(c) (i) (2) (2)}

Corela - cognition, représentation, langage est mis à disposition selon les termes de la licence Creative Commons Attribution - Pas d'Utilisation Commerciale - Partage dans les Mêmes Conditions 4.0 International. 


\title{
Test : La sous-spécification flexionnelle des bases de dérivation en question à la lumière de la morphologie bretonne
}

\author{
Pierre-Yves Kersulec
}

\section{Introduction}

\subsection{Présentation}

1 La caractérisation des unités de base des processus morphologiques, sur un plan syntaxique et sémantique, a fait l'objet d'études récentes, dans le domaine de la morphologie française notamment. Depuis Matthews (1974), les morphologues s'accordent à décrire les unités des processus morphologiques comme unités lexématiques. Une distinction est ainsi proposée entre mot phonologique, mot grammatical et unité abstraite ou lexème. $\mathrm{Si}$, d'un point de vue sémantique, le lexème, défini comme unité multidimensionnelle, est décrit comme entité linguistique pleinement spécifiée, il n'en va pas de même d'un point de vue morphosyntaxique. Les lexèmes sont en effet des unités sous-spécifiées, par définition, sur un plan flexionnel (Fradin et Kerleroux, $2008: 81)^{1}$.

2 Un certain nombre de données issues de la morphologie bretonne peuvent paraître contradictoires, à première vue, avec le principe universel proposé par Greenberg (1963: 83) selon lequel les mécanismes dérivationnels précèdent les mécanismes flexionnels, et non l'inverse. Pour Stump $(1989,1990)$, les faits bretons sont bel et bien contradictoires avec ce principe et imposent de considérer qu'un mot puisse être mobilisé comme base dérivationnelle. Acquaviva (2008) considère quant à lui que cette particularité concerne essentiellement la flexion plurielle et s'explique par l'idiosyncrasie sémantique de cette dernière. Belder (2010) estime pour sa part que la 
flexion ne précède pas la dérivation en breton, le PL1 d'une séquence $\left.\left[\mathrm{PL}_{1}\right] \mathrm{PL}_{2}\right] \mathrm{PL}$ ayant la valeur d'un allomorphe thématique, et non d'un véritable suffixe.

3 L'objectif qui est le nôtre dans cet article est avant tout de réévaluer ces différentes hypothèses à la lumière de faits nouveaux. Nous verrons en effet que les bases des processus morphologiques peuvent être porteurs d'un marquage morphologique autre que pluriel en breton. Des participes passés ou des noms féminins peuvent en effet être pris, en breton, comme opérandes, sous leur forme pleine, de suffixes flexionnels ou dérivationnels.

\subsection{Analyses antérieures}

\subsubsection{Stump $(1989 ; 1990)$}

4 Stump (1990) soutient l'hypothèse que des procédés dérivationnels peuvent, en breton, s'appliquer à des noms dotés d'une marque flexionnelle régulière. Ces faits vont à l'encontre de l'hypothèse d'une morphologie divisée entre flexion et dérivation, soutenue par Anderson (1982), et amendée par Perlmutter (1988) qui suppose que les formes flexionnelles irrégulières sont inscrites dans le lexique, ce qu'Anderson ne prévoit pas. Il est à noter cependant qu'Anderson (1982) évoque la possibilité d'insertion d'un matériau flexionnel à l' «intérieur » d'un matériau dérivationnel lorsqu'un thème est modifié par apophonie ou métaphonie ou en cas d'insertion de « véritables infixes ${ }^{2}$ ».

5 L'amendement proposé par Perlmutter à la théorie d'Anderson trouve son origine dans une interprétation de diminutifs pluriels attestés en yiddish (d'après Bochner 1984 : 415, cf. 1 a-b-c). Pour Perlmutter (1988) en effet, ces diminutifs pluriels ne sont attestés que lorsque le nom de base possède un pluriel irrégulier. Lorsque le nom singulier connaît un pluriel régulier en -en ou en $-s$ (pour les noms s'achevant par une voyelle inaccentuée), le diminutif pluriel en -l-ex ne peut sélectionner ce pluriel régulier (cf. 1d-e-f) (d'après Perlmutter, 1988 : 83, cité par Stump, $1990: 197)$ :

Tableau 1

\begin{tabular}{|l|l|l|l|l|l|}
\hline & Singulier & $\begin{array}{l}\text { Diminutif singulier en - / ou } \\
\text {-le }\end{array}$ & Pluriel & $\begin{array}{l}\text { Diminutif pluriel en - + } \\
\text {-ex }\end{array}$ \\
\hline a & Jeune marié & xosp & xosp & xosp & xosp \\
\hline b & $\begin{array}{l}\text { Livre } \\
\text { (religieux })\end{array}$ & seyfer & seyfer & seyfer & seyfer \\
\hline c & péché & xet & xet & xet & xet \\
\hline d & oreille & oyer & oyer & oyer & oyer \\
\hline e & avis & eyce & eyce & eyce & eyce \\
\hline f & cadeau & matone & matone & matone & matone \\
\hline
\end{tabular}


Pour Stump (1990), cet amendement ne permet pas de rendre compte des faits bretons qui échappent à l'hypothèse d'une morphologie divisée. Les diminutifs pluriels, relevés par Stump à Plougastel (29) mettent en effet en jeu des noms singuliers connaissant un pluriel régulier et productif, et non irrégulier (cf. infra pour d'autres exemples) :

Tableau 2

\begin{tabular}{|l|l|l|l|l|l|}
\hline & Singulier & pluriel & $\begin{array}{l}\text { Diminutif } \\
\text { singulier }\end{array}$ & $\begin{array}{l}\text { Pluriel du diminutif du } \\
\text { singulier }\end{array}$ & $\begin{array}{l}\text { Diminutif pluriel du } \\
\text { pluriel }\end{array}$ \\
\hline bateau & bag & bag-où & bagig & *bagigoù & bagoù-ig-où \\
\hline
\end{tabular}

7 Deux hypothèses sont possibles, nous dit Stump, pour rendre compte de ces diminutifs pluriels. La première d'entre elles consiste à considérer que la morphologie plurielle est dérivationnelle et non flexionnelle en breton. Le fait que cette dernière soit productive, régulière sémantiquement, et sensible aux variations syntaxiques invite en effet à rejeter cette hypothèse ${ }^{3}$. La deuxième hypothèse revient à considérer que la morphologie diminutive n'est pas véritablement dérivationnelle en breton.

8 Le suffixe -ig du diminutif ne semble en effet pas être sensible à la catégorie lexicale de la base qu'il sélectionne, contrairement aux suffixes prototypiques. Par ailleurs, il ne modifie pas la catégorie de ces bases, à l'inverse des suffixes prototypiques. Stump montre cependant que le suffixe -ig ne ressemble pas non plus aux suffixes flexionnels puisqu'il n'exprime pas de trait flexionnel pertinent pour la syntaxe, qu'il n'est pas entièrement productif et enfin qu'il n'est pas totalement régulier sur un plan sémantique.

9 Pour Stump, ces faits démontrent que les processus flexionnels peuvent être antérieurs à des processus dérivationnels. Cet auteur se refuse cependant à adopter le point de vue de Di Sciullo et Williams (1987) qui vont jusqu'à mettre en cause la distinction entre flexion et dérivation elle-même. La proposition de Stump est d'amender l'approche courante de la morphologie dérivationnelle, permettant de convertir un premier radical en un second radical (chaque radical étant associé à un paradigme distinct) (cf. 3-a). Les faits bretons incitent en effet à concevoir que la morphologie dérivationnelle puisse permettre, dans certains cas, de convertir un mot en radical (cf. 3-b) :

Tableau 3

\begin{tabular}{|l|l|l|l|}
\hline & \multicolumn{3}{|l|}{ Morphologie dérivationnelle } \\
\hline & Radical & $>$ & Radical \\
\hline & $\begin{array}{l}<\text { del }> \\
\text { del-ienn } \\
\text { feuille }\end{array}$ & & $\begin{array}{l}<\text { delienn> } \\
\text { del-ienn-où } \\
\text { feuilles }\end{array}$ \\
\hline b. & Mot & $>$ & Radical \\
\hline
\end{tabular}




\begin{tabular}{|c|c|}
\hline$<$ delioù > & <deliaouek> \\
\hline del-ioù & del-iaou-ek \\
\hline feuilles & feuillu \\
\hline
\end{tabular}

Lorsque la base de dérivation est dénuée de toute marque flexionnelle, le mot considéré peut se voir attribuer, pour cet auteur, le statut d'un radical (1990:209-210). C'est le cas des noms dénués de tout affixe permettant de créer des adjectifs en -ek par exemple (4$a-b-c)$. Dans certains cas cependant, la base peut également être un mot fléchi (4-d) :

Tableau 4

\begin{tabular}{|c|c|c|}
\hline & Base dérivationnelle & Adjectif dérivé \\
\hline a. & $\begin{array}{l}\text { Dour } \\
\text { eau }\end{array}$ & $\begin{array}{l}\text { Dourek } \\
\text { abondant en eau }\end{array}$ \\
\hline b. & $\begin{array}{l}\text { Blev } \\
\text { cheveux }\end{array}$ & $\begin{array}{l}\text { Blevek } \\
\text { chevelu }\end{array}$ \\
\hline c. & $\begin{array}{l}\text { Kof } \\
\text { ventre }\end{array}$ & $\begin{array}{l}\text { Kofek } \\
\text { ventru }\end{array}$ \\
\hline d. & $\begin{array}{l}\text { Delioù } \\
\text { feuilles }\end{array}$ & $\begin{array}{l}\text { Deliaouek } \\
\text { feuillu }\end{array}$ \\
\hline e. & Gouizi- & $\begin{array}{l}\text { Gouiziek } \\
\text { savant }\end{array}$ \\
\hline
\end{tabular}

11 Il y aurait lieu par conséquent, nous dit Stump, de poser que la suffixation en -ek peut être de type Radical > Radical ou Mot > Radical, sauf à supposer que les formes nues (4$a-b-c)$ puissent être caractérisées comme mots identiques à leurs radicaux. Les radicaux non indépendants tels que (4-e) ne peuvent se voir attribuer le statut de mots cependant, en tant que formes dépendantes. Il ressort que la suffixation en -ek peut à la fois relever de procédés de type Radical > Radical et Mot > Radical.

12 Stump fait par ailleurs l'hypothèse que « tout procédé morphologique qui s'applique à des mots de quelque catégorie peut s'appliquer également à des radicaux de cette catégorie » (Stump, 1990: 211). Cette hypothèse le conduit à postuler qu'une base marquée, sur un plan flexionnel, ne pourra être sélectionnée que s'il peut être ellemême rattachée à un processus de type Mot > Radical. Dans certains cas cependant, le mot originel peut être devenu opaque (c'est le cas de boutaoui à inconnu à Plougastel, d'après Stump, mais connu dans d'autres dialectes) :

Tableau 5

\begin{tabular}{|l|l|l|l|l|}
\hline & Mot & Radical & Mot & Mot \\
\hline
\end{tabular}




\begin{tabular}{|l|l|l|l|l|}
\hline & Nom pluriel & Radical & Verbe dérivé & Nom d'agent dérivé \\
\hline a. & $\begin{array}{l}\text { Merc'hed } \\
\text { filles }\end{array}$ & Merc'het-4 & $\begin{array}{l}\text { Merc'heta } \\
\text { courir les filles }\end{array}$ & $\begin{array}{l}\text { Merc'hetaer } \\
\text { coureur de filles }\end{array}$ \\
\hline b. & $\begin{array}{l}\text { Boutoù } \\
\text { chaussures }\end{array}$ & Boutaou- & $\begin{array}{l}\text { *Boutaoui } \\
\text { chausser, botter }\end{array}$ & $\begin{array}{l}\text { Boutaouer } \\
\text { chausseur, bottier }\end{array}$ \\
\hline
\end{tabular}

13 Stump propose également de réviser la description classique de la morphologique flexionnelle en postulant la possibilité d'une flexion de type $\operatorname{Mot}_{1}>\operatorname{Mot}_{2}$ (le mot dérivé appartenant au paradigme associé au mot de base) :

Tableau 6

\begin{tabular}{|c|c|c|c|}
\hline & \multicolumn{3}{|c|}{ Morphologie flexionnelle } \\
\hline & Radical & $>$ & Mot \\
\hline a. & $\begin{array}{l}\text { <delienn> } \\
\text { del-ienn-où } \\
\text { feuilles }\end{array}$ & & $\begin{array}{l}\text { <deliennoù> } \\
\text { del-ienn-où } \\
\text { feuilles }\end{array}$ \\
\hline b. & $\operatorname{Mot}_{1}$ & $>$ & $\mathrm{Mot}_{2}$ \\
\hline & $\begin{array}{l}\text { <bugale> } \\
\text { bugal-e } \\
\text { enfants }\end{array}$ & & $\begin{array}{l}\text { <bugaleoù> } \\
\text { bug-al-e-où } \\
\text { enfants + nuance différente }\end{array}$ \\
\hline
\end{tabular}

Ici aussi, nous dit Stump, ces deux types de procédés peuvent être intersectifs (le double pluriel bouzelleier est ainsi dérivé du pluriel simple bouzelloù lui-même rattachable au radical dépendant bouzell).

\subsubsection{Acquaviva (2008)}

15 Pour Acquaviva, l'approche correcte, pour rendre compte des faits bretons, est une approche selon laquelle les opérations grammaticales doivent être différenciées de leur réalisation ${ }^{5}$. Dans ce cadre, les processus inflexionnels et dérivationnels restent différenciés quand bien même leur réalisation peut, dans certains cas, être identique.

Cet auteur défend l'idée que les pluriels bretons sont à la fois flexionnels et dérivationnels, de manière non-ambiguë. Plusieurs arguments sont avancés par Acquaviva pour défendre le fait que les suffixes pluriels bretons ne sont pas des suffixes flexionnels prototypiques:

- Les pluriels bretons sont sensibles à la sémantique lexicale, ce qui les rapproche des suffixes dérivationnels. Les suffixes -ed et -où seraient ainsi les suffixes par défaut des animés et des inanimés, respectivement (Acquaviva, $2008: 238$ ).

- Un même nom peut également sélectionner différents suffixes pluriels sur des critères sémantiques (selon que le référent de ce dernier soit perçu comme individu discret, unité non discrétisable ou masse) 
- Le sémantisme de certains suffixes pluriels peut connaître un sémantisme idiosyncrasique (diminutif, péjoratif, duel)

- Les affixes pluriels peuvent être insérés dans un matériau non inflexionnel

(verbal, nominal, adjectival) de même que les diminutifs

17 Cette caractérisation des pluriels bretons remet en cause de façon claire la notion de lexème. Pour cet auteur, ce n'est pas tant la distinction entre flexion et dérivation qui demande à être revisitée, en breton, que le fait que ces deux types de procédés ou d'opérations morphologiques puissent connaître une même réalisation, tout en restant distincts (Acquaviva, $2008: 235$ ).

Acquaviva remet par ailleurs en cause l'affirmation de Stump selon laquelle le breton permet de sélectionner des formes fléchies comme bases des processus morphologiques. Cette possibilité relèverait en effet spécifiquement de la morphologie plurielle, et ne saurait valoir pour les autres processus flexionnels (Acquaviva, 2008 : 242). De rares exceptions seraient cependant possibles. Mention est ainsi faite, après Trépos (1956: 262), de la forme verbale redoublée me a vante-fa-fe en lieu et place de me a vantefe (je jetterais en l'air) ou encore de la suffixation en -enn au pronom hini 'celui/ un' (hiniennoù 'quelques uns'). Les bases infléchies sélectionnées par les procédés morphologiques en breton seraient principalement plurielles et mettraient par ailleurs en jeu des noms et non des pronoms, ces derniers ne formant pas une catégorie lexicale.

Pour Acquaviva, l'affirmation selon laquelle le breton permet de faire appel à un matériau flexionnel au cours d'un processus de formation nominale n'est pas fausse, mais demande à être spécifiée, les marques flexionnelles plurielles en question étant d'un type particulier (Acquaviva, $2008: 243$ ).

La conclusion de cet auteur est que le nombre forme, en breton, une catégorie flexionnelle à part entière, qui accepte cependant d'être insérée dans un matériau lexématique non flexionnel. La conclusion selon laquelle le nombre breton pourrait être tantôt de nature flexionnelle, tantôt de nature dérivationnelle, est cependant indue pour Acquaviva. Le point important est en effet que les morphèmes pluriels conservent une valeur plurielle, sur un plan sémantique, quand bien même ils se trouvent intégrés dans des bases dérivationnelles. L'idée est donc qu'un même exposant morphologique, en l'occurrence celui du nombre pluriel, peut instancier une information grammaticale aussi bien qu'une information lexicale portant sur la structuration interne d'un lexème donné.

\subsubsection{Discussion}

21 L'affirmation selon laquelle la sélection d'une base fléchie par un processus morphologique ne concernerait que les bases relevant de la flexion plurielle nous paraît pouvoir être remise en cause. Nous verrons en effet dans la deuxième partie de cet article qu'un item portant le suffixe -et du participe passé peut être sélectionné par divers procédés morphologiques en breton. Un suffixe porteur d'une information morphosyntaxique relevant du genre féminin peut également, dans certains cas, sélectionner une base déjà dotée de ce trait morphosyntaxique. 


\subsubsection{Belder (2010)}

Belder (2010) soutient que le pluriel (en l'occurrence le suffixe -ed) n'aurait pas le statut de morphème flexionnel en breton, mais un simple statut allormorphique. Cette solution aurait le mérite de préserver le principe établi dans la littérature selon lequel un suffixe flexionnel ne peut précéder un suffixe dérivationnel. Belder revient tout d'abord sur les faits suivants :

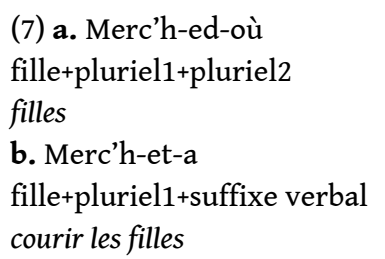

Pour Stump, ces faits remettent en cause, comme il a été vu, l'Elsewhere Principle formulé par Anderson (1986), selon lequel la dérivation précède l'inflexion. Belder soutient qu'Acquaviva refuse d'analyser le morphème PL1 (pluriel ${ }_{1}$ ) -ed- comme morphème inflexionnel doté du trait de pluralité. Cet auteur analyserait au contraire ce morphème comme morphème dérivationnel, correspondant à la réalisation d'une tête catégorielle ${ }^{6}$. Merc'hedoù répondrait ainsi, chez Acquaviva (2008), à la structure suivante, d'après Belder $(2010: 3)$ :

Schéma (1) : dérivation de « merc'hedoù » chez Acquaviva d'après Belder (2010)

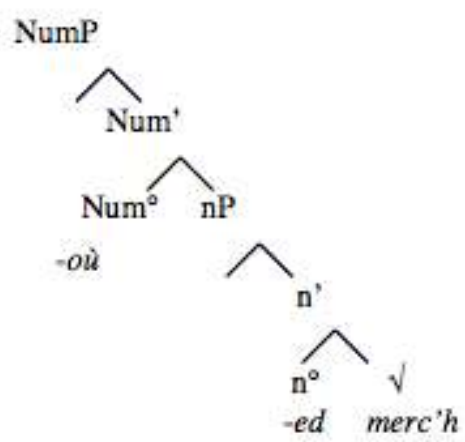

Pour Belder, cette analyse est mise en cause par le fait que les nombres cardinaux sont en distribution complémentaire avec le $\mathrm{PL}_{1}$-ed ou le $\mathrm{PL}_{2}$-où, ces derniers ayant la même réaction soumis à un cardinal ${ }^{7}$ ('ugent merc'hed 'vingt filles' est proscrit, tout comme *ugent merchedoù 'vingt filles', face au correct ugent merc'h 'vingt filles') (Belder, 2010 : 3-4). Belder montre que ce phénomène de distribution complémentaire est attesté en turc ou en hongrois également et a fait l'objet d'une interprétation par Borer (2005 : 116-117) qui propose d'assigner aux cardinaux un trait [pluriel] et un trait [\#] représentant une fonction comptable associée aux quantifieurs. Belder montre que l'interprétation proposée par Borer (que nous ne reprenons pas ici) permet de rendre compte du fait que les cardinaux réalisent une fonction [pluriel] « avant d'atteindre la tête \# ». Cette interprétation ne permettrait néanmoins pas de rendre compte du fait que les deux pluriels $\mathrm{PL}_{1}$ et $\mathrm{PL}_{2}$ soient en distribution complémentaire avec les cardinaux.

Belder soutient que la caractérisation du $\mathrm{PL}_{1}$ comme «réalisation d'une tête inflexionnelle ou dérivationnelle» conduit à une impasse. L'idée proposée par cet 
auteur pour résoudre ce problème est de supposer que les cardinaux n'entrent pas en distribution complémentaire avec le pluriel, le simple pluriel « occupant une position identique à celle du cardinal ». Pour Belder, le $\mathrm{PL}_{2}$ peut être réalisé en breton par le morphème -où ou par un morphème zéro qui entrent en compétition pour être « insérés sous le noeud réalisant le marquage du nombre » (Belder, $2010: 6-7$ ). Pour cet auteur, les noms pluriels merched ou merchedoù, auxquels elle attribue une même traduction (cf supra pour une discussion), répondent par conséquent à une structure profonde de même nature :

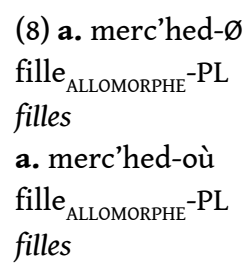

Le dérivation de ces noms pluriels est représentée comme suit par cet auteur :

Schéma (2) : dérivation de « merc'hed » et de « merc'hedoù » d'après Belder (2010)

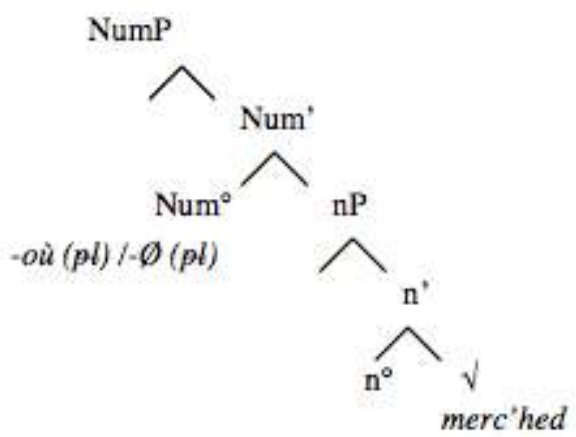

(d'après Belder, 2010:8)

Belder défend le point de vue que le simple pluriel $\mathrm{PL}_{1}$ n'est qu'un thème allomorphique sur la base de trois arguments. Le premier de ces arguments est que que ce thème -edpeut précéder le morphème pluriel -où mais également des morphèmes dérivationnels devant lesquels il peut « recouvrir une interprétation non-plurielle» (Belder, $2010: 8$ ). L'exemple steredenn est proposé par Belder pour justifier le fait que le morphème -edpourrait être dénué d'un sens pluriel. Le deuxième argument est que le morphème -edprécéderait toujours des suffixes dérivationnels. Le dernier argument proposé par Belder pour justifier le fait que le $\mathrm{PL}_{1}$ n'aurait qu'un statut d'allomorphe s'appuie sur les doubles pluriels construits sur un thème infléchi (ablaut) (e.g. louarn 'renard' > lern '(des) renards' > lerned '(des) renards').

Cette argumentation conduit Belder à affirmer que «la flexion ne précède pas la dérivation en breton ", le morphème du $\mathrm{PL}_{1}$ n'ayant pas la valeur d'un suffixe pluriel mais de « réflexe d'un pluriel plus haut » (Belder, 2010 : 9). 


\subsubsection{Discussion}

\subsection{Spécification sémantique du double pluriel} même traduction pour merc'hed et merc'hedoù à savoir girls 'filles'. Trépos ([1956] 1982 : 264) souligne de manière explicite le fait que les doubles pluriels peuvent recouvrir un sémantisme spécifique, variable selon les dialectes. Trépos rappelle ainsi qu'Ernault (1897) traduit pôtredo (double pluriel de paotr 'garçon, homme') par «beaucoup de garçons ", bugaleou (double pluriel de bugel 'enfant') par " plusieurs bandes d'enfants " et merc'hedou ou merc'hejou par "des filles $»^{8}$. En sud-cornouaillais en revanche, merc'hejou (var. merc'hijou, meryedou) ${ }^{9}$ possède, nous dit Trépos, une «nuance de mépris affectueux » ou encore parfois une valeur « diminutive et hypocoristique » rappelant le gallois merchetos ${ }^{10}$. Acquaviva rapporte que, pour Yvon Gourmelon, merch-ed-où ne serait employé que comme vocatif figé, doté d'une nuance péjorative (Acquaviva, 2008 : 260).

Il est remarquable de noter que ces différents effets de sens se laissent répartir en trois pôles distincts qui rejoignent la tripartition proposée par Fradin (2003b) pour rendre compte de l'organisation de la catégorie du diminutif. L'apport sémantique de la suffixation peut en effet être de nature référentielle ou à l'inverse évaluative. L'emploi $\mathrm{du}$ double pluriel au vocatif relève quant à lui de l'interaction du locuteur avec son interlocuteur ${ }^{11}$. Nous verrons ci-après que le redoublement d'une marque morphologique relevant de la flexion en genre peut être de nature évaluative également :

Tableau 8
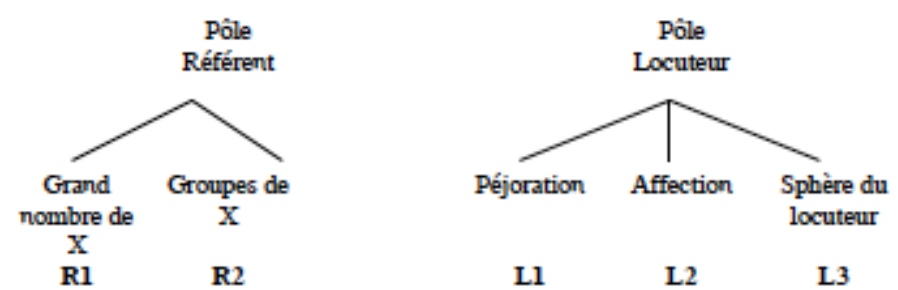

L1

L2

L3

\subsection{Caractérisation du $\mathrm{PL}_{1}$ sur le plan du nombre}

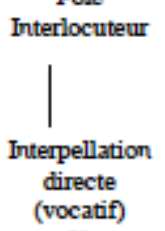

Il

La caractérisation du $\mathrm{PL}_{1}$ sur le plan du nombre - dans les séquences de type $\left[\left[\mathrm{PL}_{1}\right] \mathrm{PL}_{2}\right] \mathrm{PL}$-, demande également à être précisée. Rappelons que, pour Trépos, les noms collectifs se caractérisent par le fait qu'ils sont perçus par les bretonnants comme noms dénotant un domaine entouré d'une enveloppe ne permettant pas de distinguer les unités contenues dans ce domaine. Pour Trépos, si les noms perçus comme collectifs sont très souvent monomorphémiques et dénués de suffixe externe - tud 'gens'; per 
'poires' - un nom peut parfois être perçu comme collectif en breton quand bien même il contient un suffixe externe. Les exemples de roñsed 'chevaux' et de preñved 'vers' sont ainsi mentionnés par cet auteur. Le fait que le premier de ces noms soit construit sur une base non transparente dans certains dialectes ${ }^{12}(*[$ roñs-e $])$ rend possible, nous dit Trépos, une interprétation collective. Pour preñved, l'argument est en revanche sémantique : le nom construit renverrait à un " grouillement confus de vers $»^{13}$. Le fait que les unités dénommées ne soient pas perçues comme unités discrètes, clairement identifiables, aurait donc pour conséquence de favoriser une interprétation collective du domaine dénoté ${ }^{14}$ :

Tableau 9

\begin{tabular}{|c|c|c|c|c|c|}
\hline & $\begin{array}{l}\text { Nom } \\
\text { singulier }\end{array}$ & $\begin{array}{l}\text { Nom collectif } \\
\text { non marqué }\end{array}$ & $\begin{array}{l}\text { Nom collectif } \\
\text { marqué }\end{array}$ & $\begin{array}{l}\text { Nom pluriel marqué à } \\
\text { lecture collective }\end{array}$ & Double pluriel \\
\hline a. & $\begin{array}{l}\text { Merc'h } \\
\text { fille }\end{array}$ & - & - & $\begin{array}{l}\text { Merc'hed } \\
\text { filles et/ou femmes }\end{array}$ & $\begin{array}{l}\text { Merc'hed-où } \\
\text { Id. + spéc. sém. }\end{array}$ \\
\hline b. & $\begin{array}{l}\text { Paotr } \\
\text { garçon }\end{array}$ & - & - & $\begin{array}{l}\text { Paotred } \\
\text { garçons et/ou hommes }\end{array}$ & $\begin{array}{l}\text { Paotred-où } \\
\text { id. + spéc. sém. }\end{array}$ \\
\hline c. & $\begin{array}{l}\text { Bugel } \\
\text { enfant }\end{array}$ & - & - & $\begin{array}{l}\text { Bug-al-e } \\
\text { enfants }\end{array}$ & $\begin{array}{l}\text { Bug-al-e-où } \\
\text { id. + spéc. sém. }\end{array}$ \\
\hline d. & $\begin{array}{l}\text { Den } \\
\text { personne }\end{array}$ & $\begin{array}{l}\text { Tud } \\
\text { gens }\end{array}$ & - & - & $\begin{array}{l}\text { Tud-où } \\
\text { id. + spéc. sém. }\end{array}$ \\
\hline e. & - & $\begin{array}{l}\text { Dilhad } \\
\text { habits }\end{array}$ & - & - & $\begin{array}{l}\text { Dilhad-où } \\
\text { id. + spéc. sém. }\end{array}$ \\
\hline f. & $\begin{array}{l}\text { Bruzhanenn } \\
\text { miette }\end{array}$ & $\begin{array}{l}\text { Bruzhan } \\
\text { miettes }\end{array}$ & $\begin{array}{l}\text { Bruzhan-aj } \\
\text { Id. + spéc. sém.. }\end{array}$ & - & $\begin{array}{l}\text { Bruhan-où, } \\
\text { Inguiniel- } \\
\text { Id. + spéc. sém. }\end{array}$ \\
\hline g. & - & - & $\begin{array}{l}\mathrm{Glep}_{\mathrm{ADJ}}{ }^{-\mathrm{aj}} \\
\text { Propos/actes } \\
\text { idiots }\end{array}$ & - & $\begin{array}{l}\text { Glep-aj-où, } \\
\text { Inguiniel- } \\
\text { id. + spéc. sém }\end{array}$ \\
\hline
\end{tabular}

(D’après Trépos, 1982, passim, et des données recueillies personnellement)

Lorsque le suffixe -où est le premier membre du double pluriel, dans la séquence [[-où]ier -ioù]PL ${ }^{15}$, la situation peut être différente. Le PL1 en -où peut avoir valeur de duel ou de collectif (interprétation défendue par Acquaviva, 2008). Il peut également, en revanche, dénoter également un pluriel discontinu ou discrétisant (10-f). Le suffixe oùier peut également être perçu dans certains cas, par analogie, comme suffixe autonome, comme le soulignait déjà Trépos (1982) à propos des dérivés glav-eier ou dour-eier 'grandes pluies' formés sur des noms singuliers. A la différence de ces dérivés toutefois, qui possèdent un $\mathrm{N}$-ioù discontinu correspondant, le double pluriel zaoutoùier ne peut être rapporté au pluriel *zaout-où non-attesté : 
Tableau10

\begin{tabular}{|c|c|c|c|c|c|}
\hline & $\begin{array}{l}\text { Nom } \\
\text { singulier }\end{array}$ & $\begin{array}{l}\text { Nom } \\
\text { pluriel en - } \\
\text { où }\end{array}$ & Nom duel en -où & $\begin{array}{l}\text { Nom } \\
\text { collectif }\end{array}$ & Double pluriel \\
\hline a. & $\begin{array}{l}*[\text { Brag }]-\text { où } \\
\text { pantalon }\end{array}$ & - & $\begin{array}{l}*[\text { Brag }] \text {-où } \\
\text { pantalon (une } \\
\text { paire de jambes) }\end{array}$ & - & $\begin{array}{l}*[\text { Brag] }] \text {-eier } \\
\text { plusieurs paires de X }\end{array}$ \\
\hline b. & $\begin{array}{l}*[\mathrm{Ot}]-\text { aou- } \\
\text { enn } \\
\text { jambe de } \\
\text { pantalon }\end{array}$ & - & $\begin{array}{l}*[\mathrm{Ot}] \text {-où } \\
\text { pantalon (une } \\
\text { paire de jambes) }\end{array}$ & - & $\begin{array}{l}*[\mathrm{Ot}] \text {-eier } \\
\text { plusieurs paires de } X\end{array}$ \\
\hline c. & $\begin{array}{l}\text { Louz-aou- } \\
\text { enn } \\
\text { herbe } \\
\text { médicinale }\end{array}$ & - & - & $\begin{array}{l}\text { Louz-où } \\
\text { herbes } \\
\text { médicinales }\end{array}$ & $\begin{array}{l}\text { Louz-eier } \\
\text { nbre indéterminé de } \mathrm{X}\end{array}$ \\
\hline d. & $\begin{array}{l}*[\text { Goul }] \text {-où } \\
\text { lumière } \\
*[\text { Goul }] \text {-aou- } \\
\text { enn } \\
\text { cierge }\end{array}$ & - & - & $\begin{array}{l}*[\text { Goul }] \text {-où } \\
\text { lumières }\end{array}$ & $\begin{array}{l}*[\text { Goul }] \text {-eier } \\
\text { nbre indéterminé de X }\end{array}$ \\
\hline e. & $\begin{array}{l}\square[\text { Bot }] \mathrm{ez}^{16} \\
\text { chaussure }\end{array}$ & - & $\begin{array}{l}\square[\text { Bot }] \text {-où } \\
\text { chaussures (paire) }\end{array}$ & - & $\begin{array}{l}\square[\text { Bot }] \text {-o-ioù } \\
\text { nbre indéterminé de } X\end{array}$ \\
\hline f. & $\begin{array}{l}\text { Park } \\
\text { champ }\end{array}$ & $\begin{array}{l}\text { Park-où } \\
\text { champs }\end{array}$ & - & - & $\begin{array}{l}\text { Park-eier } \\
\text { nbre indéterminé de X }\end{array}$ \\
\hline g. & $\begin{array}{l}\text { Buoc'h } \\
\text { vache }\end{array}$ & - & - & $\begin{array}{l}\text { Zaout } \\
\text { vaches }\end{array}$ & $\begin{array}{l}\text { Zaout-où-ier, -Berné 56- } \\
\text { Ensemble hétérogène, de nbre } \\
\text { indéterminé, de vaches + (veaux + } \\
\text { génisses) }\end{array}$ \\
\hline
\end{tabular}

(D’après Trépos, 1982, passim, et des données recueillies personnellement)

Pour Acquaviva, il conviendrait d'être prudent devant les résultats proposés par Trépos, ces derniers étant issus de divers dialectes. Les « repluralisations » en -où+-ier ne formeraient pas en réalité un procédé morphologique régulier (Acquaviva, 2008 : 255). Acquaviva s'appuie sur les fait que plusieurs doubles pluriels en -eier mentionnés par Trépos (1982: 232) ont en réalité été refusés par les informateurs qu'il a consultés. La forme parkeier serait par ailleurs devenue le pluriel courant de park en centre Bretagne (Wmffre, 1998 : 14).

Nous relevons nous-même cette lexicalisation de parkeier à Melgven (29) où le pluriel discontinu parkoù est également inusité. Différents locuteurs vannetais que nous avons 
consultés nous ont en revanche confirmé l'opposition régulière qu'ils perçoivent entre les doubles pluriels en -où-ier et les pluriels en -où correspondants :

(11) a. Parkoùier zo ur yeuc'h. $\mathrm{O}$, bout 'eus ur yeuc'h parkoùier.

n-oùier+est+un tas. Oh, +être+il y a+un tas+n-oùier

'Parkoùier' c'est un tas. Oh, il y a un tas de 'parkoùier'.

a'. Ha parkoù 'veze ket nameit un nebeudig. Un nebed. Revet: pemp pe c'hwec'h park.

et+n-où+n'était que (d'habitude)+un+petit peu. Un peu. Ca dépend: cinq+ou+six+champ.

Et 'parkoù' ça n'était qu'un petit peu. Un peu. Ca dépend : cinq ou six champs.

a". Ha pa veze ur yeuc'h èl-se : o, bout 'eus ur yeuc'h parkoùier, -St Barthélémy, 56-

et+quand+il y avait (d'habitude)+un tas+comme ça : oh, être+il y a+un tas+n-oùier

Et quand il y en avait un tas comme ça: oh, il y a un tas de champs

Nous relevons notamment les doubles pluriels suivants ${ }^{17}$ auprès de divers informateurs ${ }^{18}$ :

Tableau 12

\begin{tabular}{|c|c|c|c|}
\hline & Nom singulier & Pluriel en -où & Pluriel en -oùier \\
\hline a. & $\begin{array}{l}\text { Lann } \\
\text { lande }\end{array}$ & Lann-où & Lann-où-ier, -St Barthélémy, 56- \\
\hline b. & $\begin{array}{l}\text { Koed } \\
\text { bois }\end{array}$ & Koed-où & Koed-où-ier, -St Barthélémy, 56- \\
\hline c. & $\begin{array}{l}\text { Douar } \\
\text { terre }\end{array}$ & Douar-où & Douar-où-ier, -St Barthélémy, 56- \\
\hline d. & $\begin{array}{l}\text { Forn } \\
\text { four }\end{array}$ & Forn-où & Forn-où-ier, -St Barthélémy, 56- \\
\hline e. & $\begin{array}{l}\text { Gwaezh } \\
\text { ruisseau }\end{array}$ & Gwaezh-où & Gwaezh-oùi-ier, -Séglien, 56- \\
\hline f. & $\begin{array}{l}\text { Feurm } \\
\text { ferme }\end{array}$ & Feurm-où & Feurm-où-ier, -Berné, 56- \\
\hline g. & $\begin{array}{l}\text { Pioch } \\
\text { pioche }\end{array}$ & Pioch-où & Pioch-où-ier, -Berné, 56- \\
\hline h. & $\begin{array}{l}\text { Ivarzh } \\
\text { voie charretière }\end{array}$ & Ivarzh-où & Ivarzh-où-ier, -Carnac, 56- \\
\hline
\end{tabular}

D’après Trépos (1982 : 66)

34 L'on aboutit par conséquent à trois structures différentes - la troisième d'entre elles n'étant attestée que de manière hapaxique, dans quelques points du Trégor uniquement vraisemblablement - :

1. [[nom collectif/duel/pluriel à valeur collective] [pluriel discontinu en -où] double pluriel 
2. [[pluriel discontinu en -où] [pluriel discontinu en -ier] double pluriel

3. [[duel en -où] [pluriel discontinu en -où] double pluriel Acquaviva (2008) notamment. Rappelons que Trépos (1982: 274-275) fait l'hypothèse que l'usage de ces pluriels semble avoir été généralisé en breton littéraire par Grégoire de Rostrenen ${ }^{19}$. Les pluriels en -où-igoù seraient cependant exceptionnels en SudCornouaille notamment. Trépos fait l'hypothèse que ces diminutifs sont nés par souci d' «évoquer immédiatement l'image du pluriel » dans le noms dérivés. Le grammairien propose par ailleurs un parallèle entre ces pluriels et les pluriels discrets en -enn-où (suffixe du singulatif + suffixe pluriel) formés sur un nom pluriel/collectif ${ }^{20}$. Dans les deux cas en effet, un suffixe diminutif/singulatif est intercalé entre deux suffixes pluriels ${ }^{21}$. Trépos précise par ailleurs que la règle voudrait, dans certaines grammaires, que le diminutif peut connaître un pluriel simple lorsqu'il n'est pas formé sur un substantif (eg. brav ${ }_{\mathrm{ADJ}}$ 'beau' > bravigoù ${ }_{\mathrm{NPL}}$ 'jouets').

Les différents types de diminutifs pluriels relevés par Stump dans le parler de Plougastel (29) sont les suivants :

Tableau 13

\begin{tabular}{|c|c|c|c|c|}
\hline a. & Singulier & Diminutif singulier & Pluriel & Diminutif pluriel \\
\hline 1. & $\begin{array}{l}\text { Bag } \\
\text { bateau }\end{array}$ & $\begin{array}{l}\text { Bagig } \\
\text { petit bateau }\end{array}$ & $\begin{array}{l}\text { Bagoù } \\
\text { (des) bateaux }\end{array}$ & $\begin{array}{l}\text { Bagoùigoù } \\
\text { (des) petits bateaux }\end{array}$ \\
\hline 2. & $\begin{array}{l}\text { Pedenn } \\
\text { prière }\end{array}$ & $\begin{array}{l}\text { Pedennig } \\
\text { petite prière }\end{array}$ & $\begin{array}{l}\text { Pedennoù } \\
\text { (des) prières }\end{array}$ & $\begin{array}{l}\text { Pedennoùigoù } \\
(\text { des }) \text { petites prières }\end{array}$ \\
\hline 3. & $\begin{array}{l}\text { tra } \\
\text { chose }\end{array}$ & $\begin{array}{l}\text { Traig } \\
\text { petite chose }\end{array}$ & $\begin{array}{l}\text { Traoù } \\
\text { (des) choses }\end{array}$ & $\begin{array}{l}\text { Traoùigoù } \\
\text { (des) petites choses }\end{array}$ \\
\hline b. & Singulier & Diminutif singulier & Pluriel en -ed & Diminutif pluriel \\
\hline
\end{tabular}

Corela, 9-1 | 2011 


\begin{tabular}{|c|c|c|c|c|}
\hline 1. & $\begin{array}{l}\text { Merc'h } \\
\text { fille }\end{array}$ & $\begin{array}{l}\text { Merc'hig } \\
\text { petite fille }\end{array}$ & $\begin{array}{l}\text { Merc'hed } \\
\text { (des) filles }\end{array}$ & $\begin{array}{l}\text { Merc'hedigoù } \\
\text { (des) petites filles }\end{array}$ \\
\hline 2. & $\begin{array}{l}\text { Paotr } \\
\text { garçon }\end{array}$ & $\begin{array}{l}\text { Paotrig } \\
\text { petit garçon }\end{array}$ & $\begin{array}{l}\text { Paotred } \\
\text { (des) garçons }\end{array}$ & $\begin{array}{l}\text { Paotredigoù } \\
\text { (des) petits garçons }\end{array}$ \\
\hline c. & Singulier & Diminutif singulier & Inflexion $-e l->-a l+$ pluriel en $-e$ & Diminutif pluriel \\
\hline & $\begin{array}{l}\text { Bugel } \\
\text { enfant }\end{array}$ & $\begin{array}{l}\text { Bugelig } \\
\text { petit enfant }\end{array}$ & $\begin{array}{l}\text { Bugale } \\
\text { (des) enfants }\end{array}$ & $\begin{array}{l}\text { Bugaligoù } \\
\text { (des) petits enfants }\end{array}$ \\
\hline d. & Singulier & Diminutif singulier & Pluriel en $-e z$ & Diminutif pluriel \\
\hline & $\begin{array}{l}\mathrm{Ti} \\
\text { maison }\end{array}$ & $\begin{array}{l}\text { Tiig } \\
\text { Petite maison }\end{array}$ & $\begin{array}{l}\text { Tiez } \\
\text { (des) maisons }\end{array}$ & $\begin{array}{l}\text { Tiezigoù } \\
\text { (des) petites maisons }\end{array}$ \\
\hline e. & Singulier & Diminutif singulier & Pluriel interne & Diminutif pluriel \\
\hline 1. & $\begin{array}{l}\text { Askorn } \\
\text { os }\end{array}$ & $\begin{array}{l}\text { Askornig } \\
\text { petit os }\end{array}$ & $\begin{array}{l}\text { Eskern } \\
\text { (des) os }\end{array}$ & $\begin{array}{l}\text { Eskernigoù } \\
\text { (des) petits os }\end{array}$ \\
\hline 2. & $\begin{array}{l}\text { Maen } \\
\text { pierre }\end{array}$ & $\begin{array}{l}\text { Maenig } \\
\text { petite pierre }\end{array}$ & $\begin{array}{l}\text { Mein } \\
\text { (des) pierres }\end{array}$ & $\begin{array}{l}\text { Meinigoù } \\
\text { (des) petites pierres }\end{array}$ \\
\hline f. & Singulier & Diminutif singulier & Nom collectif & Diminutif pluriel \\
\hline 1. & - & $?$ & $\begin{array}{l}\text { Dilhad } \\
\text { (ensemble de) vêtements }\end{array}$ & $\begin{array}{l}\text { Dilhadigoù } \\
\text { (des) petits vêtements }\end{array}$ \\
\hline 2. & $\begin{array}{l}\text { Den } \\
\text { homme }\end{array}$ & $\begin{array}{l}\text { Denig } \\
\text { petit homme }\end{array}$ & $\begin{array}{l}\text { Tud } \\
\text { (ensemble) de gens }\end{array}$ & $\begin{array}{l}\text { Tudigoù } \\
\text { Personnes + spéc. sém. }\end{array}$ \\
\hline
\end{tabular}

Il ressort tout d'abord que le suffixe -igoù peut sélectionner des noms collectifs non marqués ou à pluriel interne (11 e-f), mais aussi des noms marqués à valeur collective précédemment évoqués $(11 \mathrm{~b}-\mathrm{c})$. A ces derniers noms vient s'ajouter le pluriel tiez, porteur du suffixe -ez. L'on ne manque pas d'observer que ce suffixe, relativement rare, accepte la suffixation en -enn de même que le suffixe -ed, d'où une lecture collective (eg. $\square[$ patat-ez 'pommes de terre' > patatez-enn 'pomme de terre'). L'interprétation collective du $\mathrm{PL}_{1}$ fait ici difficulté en revanche dans l'exemple (11-a2) tout particulièrement, porteur d'un pluriel discrétisant en -enn-où. L'hypothèse allomorphique paraît difficilement tenable ici également par conséquent pour rendre compte de la sélection du suffixe -où comme $\mathrm{PL}_{1}$.

Remarque

Trépos (1982) évoque brièvement le fait que les pluriels simples en -igoù seraient tout autant disponibles que les pluriels complexes en -oùigoù. Une nuance sémantique existerait par ailleurs entre ces deux derniers.

41 Nous procédons ici à de brèves remarques sur ce point. Il ressort que l'étude du fonctionnement du pluriel du diminutif dans divers dialectes révèle le fait que le pluriel 
du diminutif peut être systématique, ou ne pas l'être, et entrer en variation avec un pluriel formé sur le diminutif singulier. Les données relevées varient nettement d'un informateur à l'autre. L'informateur A1 de Berné (56) refuse ainsi parfois tantôt le pluriel du diminutif singulier, tantôt celui du diminutif pluriel (12-b). Dans d'autres cas, c'est le pluriel du diminutif pluriel qui est refusé (12-c-d), là où le pluriel du diminutif singulier est accepté. Dans un troisième cas de figure, enfin, c'est le pluriel du diminutif pluriel qui est accepté, alors que le pluriel du diminutif singulier est refusé (12-e-f) :

Tableau 14

\begin{tabular}{|c|c|c|c|c|c|}
\hline & $\begin{array}{l}\text { Nom } \\
\text { singulier }\end{array}$ & Diminutif & $\begin{array}{l}\text { Pluriel du diminutif } \\
\text { singulier }\end{array}$ & Pluriel & $\begin{array}{l}\text { Pluriel du diminutif } \\
\text { pluriel }\end{array}$ \\
\hline a. & $\begin{array}{l}\text { Yar } \\
\text { poule }\end{array}$ & $\begin{array}{l}\text { ? Yarig } \\
\text { petite poule } \\
\text { Yar vihan } \\
\text { petite poule }\end{array}$ & $\begin{array}{l}\text { *Yarigoù } \\
\text { petites poules }\end{array}$ & $\begin{array}{l}\text { Yer } \\
\text { poules }\end{array}$ & $\begin{array}{l}{ }^{\circ} \text { Yerigoù }^{22} \\
\text { Petites poules }\end{array}$ \\
\hline b. & $\begin{array}{l}\text { Buoc'h } \\
\text { vache }\end{array}$ & $\begin{array}{l}\text { Buoc'hig } \\
\text { Petite vache }\end{array}$ & $\begin{array}{l}\text { *Buoc'higoù } \\
\text { petites vaches }\end{array}$ & $\begin{array}{l}\text { Zaout } \\
\text { Vaches }\end{array}$ & $\begin{array}{l}{ }^{*} \text { Zaoutigoù } \\
\\
\text { Petites vaches }\end{array}$ \\
\hline c. & $\begin{array}{l}\text { Evn } \\
\text { oiseau }\end{array}$ & $\begin{array}{l}\text { Evnig } \\
\text { Petit oiseau } \\
\text { Evn bihan } \\
\text { Petit oiseau }\end{array}$ & $\begin{array}{l}\text { Evnigoù } \\
\text { petits oiseaux } \\
\text { Evned pihan } \\
\text { petits oiseaux }\end{array}$ & $\begin{array}{l}\text { Evned } \\
\text { oiseaux }\end{array}$ & $\begin{array}{l}{ }^{*} \text { Evnedigoù } \\
\text { petits oiseaux }\end{array}$ \\
\hline d. & $\begin{array}{l}\text { Maouez } \\
\text { femme }\end{array}$ & $\begin{array}{l}\text { Maouezig } \\
\text { Petite } \\
\text { femme }\end{array}$ & $\begin{array}{l}\text { Maouezigoù } \\
\text { Petites femmes }\end{array}$ & $\begin{array}{l}\text { Maouezed } \\
\text { femmes }\end{array}$ & $\begin{array}{l}{ }^{*} \text { Maouezedigoù }{ }^{24} \\
\text { petites femmes }\end{array}$ \\
\hline e. & $\begin{array}{l}\text { Merc'h } \\
\text { fille }\end{array}$ & $\begin{array}{l}\text { Merc'hig } \\
\text { petite fille }\end{array}$ & $\begin{array}{l}\text { *Merc'higoù } \\
\text { petites filles }\end{array}$ & $\begin{array}{l}\text { Merc'hed } \\
\text { filles }\end{array}$ & $\begin{array}{l}\text { Merc'hedigoù } \\
\text { petites filles }\end{array}$ \\
\hline f. & $\begin{array}{l}\text { Paotr } \\
\text { garçon }\end{array}$ & $\begin{array}{l}\text { Paotrig } \\
\text { petit garçon }\end{array}$ & $\begin{array}{l}\text { *Paotrigoù } \\
\text { petits garçons }\end{array}$ & $\begin{array}{l}\text { Paotred } \\
\text { garçons }\end{array}$ & $\begin{array}{l}\text { Paotredigoù } \\
\text { petits garçons }\end{array}$ \\
\hline g. & $\begin{array}{l}\mathrm{Ki} \\
\text { chien }\end{array}$ & $\begin{array}{l}{ }^{*} \text { Kiig } \\
\text { petit chien }\end{array}$ & $\begin{array}{l}\text { *Kiigoù } \\
\text { petits chiens }\end{array}$ & $\begin{array}{l}\text { Chach } \\
\text { chiens }\end{array}$ & $\begin{array}{l}\text { Chachigoù }^{25} \\
\text { petits chiens }\end{array}$ \\
\hline
\end{tabular}

Il ressort par ailleurs que, dans les dialectes de Berné (56) et d'Inguiniel (56), la séquence -oùigoù n'est pas permise. La seule exception que nous connaissons est illustrée par le pluriel traoùigoù 'petites choses' aux côtés de *traigoù refusé par nos informateurs. Cette exception nous semble justifiable sur un plan morphologique (la réalisation du nom pluriel comme nom monosyllabique favorise une lecture collective de ce dernier) et sémantique (traoù renvoie à une collection hétérogène de nombre indéterminé). 


\subsection{Autonomie du suffixe -ed} ce suffixe précéderait toujours un suffixe dérivationnel, et ne pourrait pas être séparé, par conséquent, de la base à laquelle il s'adjoint ${ }^{26}$. Dans plusieurs cas cependant, un matériau dérivationnel peut bel et bien séparer le suffixe pluriel d'une base de dérivation donnée :

Tableau 15

\begin{tabular}{|c|c|c|c|}
\hline & Base de dérivation & Suffixe(s) intercalé(s) & Suffixe terminal \\
\hline a. & $\begin{array}{l}\text { Dorn-a } \tilde{n}_{\mathrm{VB}} \\
\text { battre }\end{array}$ & $\begin{array}{l}\text { Dorn-er-ez } \\
\text { batteuse }\end{array}$ & $\begin{array}{l}\text { Dorner-ez-ed } \\
\text { batteuses }\end{array}$ \\
\hline b. & $\begin{array}{l}\text { Gwenn- } \\
\text { ADJ } \\
\text { blanc }\end{array}$ & $\begin{array}{l}\text { Gwenn-ig-ell } \\
\text { Hirondelle (Fav, } 1992: 309 \mathrm{~b})\end{array}$ & $\begin{array}{l}\text { Gwenn-ig-ell-ed } \\
\text { hirondelles }\end{array}$ \\
\hline
\end{tabular}

\subsection{Bilan}

L'hypothèse allomorphique proposée par Belder (2010) vise à attribuer au $\mathrm{PL}_{1}$-ed le statut d'allomorphe thématique et non de suffixe pluriel. Cette hypothèse, si elle devait s'avérer valide, devrait rendre compte du fait que les suffixes -où ou -aj ont également un statut allomorphique. Ces suffixes peuvent en effet se voir adjoindre un autre suffixe pluriel également (-ier ou -où) et sont dotés d'un nombre syntaxique pluriel incompatible avec la détermination d'un cardinal. Ce test de détermination révèlerait en effet que le collectif glepaj 'des actes/propos idiots' refuse tout autant d'être déterminé par ugent 'vingt' que le pluriel glepaj-où, les noms collectifs - suffixés ou non - étant syntaxiquement pluriel en breton (cf. Stump, 1989). La solution la plus simple, pour éviter d'attribuer systématiquement un statut allomorphique au $\mathrm{Pl}_{1}$ issu d'une séquence $\left[\left[\mathrm{Pl}_{1}\right]\left[\mathrm{PL}_{2}\right] \mathrm{PL}\right.$ est de considérer que ce pluriel et sémantiquement marqué tout en étant syntaxiquement neutralié (statut dérivationnel).

\section{Eléments d'analyse supplémentaire}

Nous pensons que les faits qui viennent d'être discutés demandent à réévalués à la lumière de données supplémentaires ne concernant pas que la flexion plurielle exclusivement.

\subsection{La base de dérivation est un nom fléchi au participe passé}

Il ressort tout d'abord que le breton partage la possibilité avec le gallois de sélectionner des bases porteuses du suffixe -et permettant de créer des adjectifs verbaux - ou participes passés ${ }^{27}-$. Ce suffixe peut accepter les combinaisons suivantes en breton :

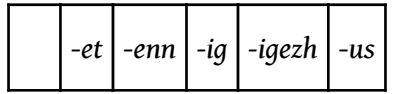




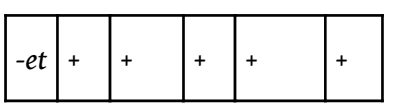

\subsubsection{Séquence -edet} peut ainsi être rapporté au participe passé ou au verbe kousket - ces deux formes étant homophones -. Kleñvedus 'morbide, pathogène' ne semble en revanche pas pouvoir être rattaché à 'kleñvediñ, non attesté chez Favereau (1992). Luc'hedus 'à éclairs' présente un autre cas de figure, les verbes luc'hedañ luc'hedin ne pouvant être rapportés à un participe passé, mais à un nom. Dans un dernier cas de figure, l'adjectif ne peut être dérivé que d'un verbe (monet 'aller' > monedus 'allant, sociable) :

Tableau 16

\begin{tabular}{|c|c|c|c|c|c|c|c|}
\hline & $\begin{array}{l}\text { Nom en } \quad \text { ed } \\
\text { dérivé } \\
\text { étymologiquemen } \\
\text { d'un participe } \\
\text { passé }\end{array}$ & $\begin{array}{l}\text { Nom } \\
\text { singulier } \\
\text { en -ed }\end{array}$ & $\begin{array}{l}\text { Nom } \\
\text { pluriel } \\
\text { en -ed }\end{array}$ & $\begin{array}{l}\text { Participe } \\
\text { passé en } \\
\text {-et }\end{array}$ & Verbe & $\begin{array}{l}\text { Adjectif } \\
\text { déverbal ou } \\
\text { désadjectival }\end{array}$ & $\begin{array}{l}\text { Nom } \\
\text { désadjectival }\end{array}$ \\
\hline a. & $\begin{array}{l}\text { Kleñved } \\
\text { maladie }\end{array}$ & - & & - & - & $\begin{array}{l}\text { Kleñvedus } \\
\text { Morbide, } \\
\text { pathogène }\end{array}$ & \\
\hline b. & - & - & $\begin{array}{l}\text { Luc'hed } \\
\text { éclairs }\end{array}$ & - & $\begin{array}{l}\text { Luc'hedañ } \\
(1521) \\
\text { Luc'hediñ } \\
(1659) \\
\text { éclairer }\end{array}$ & $\begin{array}{l}\text { Luc'hedus } \\
(1821) \\
\text { à éclairs }\end{array}$ & - \\
\hline
\end{tabular}




\begin{tabular}{|l|l|l|l|l|l|l|l|}
\hline c. & - & $\begin{array}{l}\text { Kousked } \\
\text { sommeil }\end{array}$ & - & $\begin{array}{l}\text { Kousket } \\
\text { endormi }\end{array}$ & $\begin{array}{l}\text { Kousket } \\
\text { dormir }\end{array}$ & $\begin{array}{l}\text { Kouskedus } \\
\text { endormant }\end{array}$ & $\begin{array}{l}\text { Kouskedenn } \\
\text { couchage, } \\
\text { couchette }\end{array}$ \\
\hline e. & - & - & $\begin{array}{l}\text { Loened } \\
\text { animaux }\end{array}$ & - & $\begin{array}{l}\text { Repeupler } \\
(1992)\end{array}$ & $\begin{array}{l}\text { Giboyeux } \\
(1521)\end{array}$ & - \\
\hline d. & - & - & - & - & $\begin{array}{l}\text { Monet } \\
\text { aller }\end{array}$ & $\begin{array}{l}\text { Monedus } \\
(1919) \\
\text { allant, sociable }\end{array}$ & - \\
\hline
\end{tabular}

(D’après Deshayes, 2003)

\subsubsection{Suffixes -edig et -edigezh -idigezh}

Le suffixe -edig - non productif en breton contemporain - est issu étymologiquement d'un ancien *-ātikos (Fleuriot, 1989 : 314-315). Ce suffixe attestée en vieux-breton sous la forme graphique -etic. Fleuriot (id.) note que les adjectifs en -etic se répartissent, en vieux-breton, en deux groupes. Un premier groupe regroupe des dérivés à valeur d'adjectif ou de véritables participes présents (ex. TESHEGETIC «brûlant, chaud»). Un deuxième groupe regroupe des dérivés dans lesquels la valeur de participe passé « semble nette » (ex. REGoteTIC « confié », LOSCITIC « brûlé ») (ibid.).

51 Le suffixe - eticaith ${ }^{28}$ est également attesté en vieux-breton. Il donne en breton moderne le suffixe-idigezh (vannetais -edigezh) et correspond au gallois -edigaeth (Fleuriot, 1989: 350). Il ressort, si l'on se base sur le lexique attesté, que le nom abstrait en -edigezh être rattaché à un adjectif en -edig - auquel cas l'interprétation du suffixe-edigezh comme suffixe autonome ne doit pas être privilégiée -. Il peut également être dérivé directement du participe passé d'un verbe donné (cf. 18-f-j). Une interprétation du suffixe comme suffixe autonome s'impose dès lors:

Tableau 18

\begin{tabular}{|c|c|c|c|c|}
\hline & Verbe & Participe passé & Adjectif dérivé & Nom abstrait \\
\hline a. & $\begin{array}{l}\text { Ganiñ } \sim \text { genel } \\
\text { naître }\end{array}$ & $\begin{array}{l}\text { Ganet } \\
\text { né }\end{array}$ & $\begin{array}{l}\text { Ganedig } \sim \text { ginidig } \\
\text { natif }\end{array}$ & $\begin{array}{l}\text { Ganedigezh } \\
\text { naissance }\end{array}$ \\
\hline b. & $\begin{array}{l}\text { Trec'hiñ } \\
\text { vaincre }\end{array}$ & $\begin{array}{l}\text { Trec'het } \\
\text { vaincu }\end{array}$ & $\begin{array}{l}\text { Trec'hedig } \\
\text { vaincu }\end{array}$ & $\begin{array}{l}\text { Trec'hedigezh } \\
\text { défaite }\end{array}$ \\
\hline b. & $\begin{array}{l}\text { Terriñ } \\
\text { casser }\end{array}$ & $\begin{array}{l}\text { Terret } \\
\text { cassé }\end{array}$ & $\begin{array}{l}\text { Terridig } \\
\text { Pénible }\end{array}$ & - \\
\hline c. & $\begin{array}{l}\text { Karet } \\
\text { aimer }\end{array}$ & $\begin{array}{l}\text { Karet } \\
\text { aimé }\end{array}$ & $\begin{array}{l}\text { Karedig } \\
\text { cher }\end{array}$ & - \\
\hline
\end{tabular}




\begin{tabular}{|c|c|c|c|c|}
\hline e. & $\begin{array}{l}\text { Leskel } \\
\text { brûler }\end{array}$ & $\begin{array}{l}\text { Lesket } \\
\text { brûlé }\end{array}$ & $\begin{array}{l}\text { Leskidig } \\
\text { brûlant }\end{array}$ & - \\
\hline f. & $\begin{array}{l}\text { Gortoz } \\
\text { attendre }\end{array}$ & $\begin{array}{l}\text { Gortozet } \\
\text { attendu }\end{array}$ & - & $\begin{array}{l}\text { Gortozedigezh } \\
\text { Attente désirée (Catholicon) }\end{array}$ \\
\hline g. & $\begin{array}{l}\text { Gouarn } \\
\text { garder }\end{array}$ & $\begin{array}{l}\text { Gouarn } \\
\text { garder }\end{array}$ & - & $\begin{array}{l}\text { Gouarnedigezh } \\
\text { Gardiennage de maison (GReg) }\end{array}$ \\
\hline h. & $\begin{array}{l}\text { Gwelet } \\
\text { voir }\end{array}$ & $\begin{array}{l}\text { Gwelet } \\
\text { voir }\end{array}$ & - & $\begin{array}{l}\text { Gweledigezh } \\
\text { vision, apparence (GReg) }\end{array}$ \\
\hline i. & $\begin{array}{l}\text { Kalediñ } \\
\text { durcir }\end{array}$ & $\begin{array}{l}\text { Kaledet } \\
\text { durci }\end{array}$ & - & $\begin{array}{l}\text { Kaledigezh } \\
\text { durcissement, parf. constipation }\end{array}$ \\
\hline j. & $\begin{array}{l}\text { Monet } \\
\text { aller }\end{array}$ & $\begin{array}{l}\text { Aet } \\
\text { allé }\end{array}$ & - & $\begin{array}{l}\text { Monedigezh } \\
\text { entrée (GReg) }\end{array}$ \\
\hline
\end{tabular}

(D'après Deshayes, 2003)

\subsubsection{Séquence-edenn}

52 Nous ne relevons que de rares exemples de noms en -enn construits sur un participe passé en -et. Mentionnons soubedenn 'mouillette (de pain, \& tanche à tremper T)' (Favereau, 1992: 689b) construit de manière indéniable sur le participe passé soubet 'trempé. Il n'y a pas lieu en effet ici d'évoquer une dérivation sur un infinitif homophone en -et, ce dernier n'étant pas attesté ( ${ }^{\circ}$ soubet $_{\mathrm{PP}} \mathrm{vs}^{*}{ }^{*}$ soubet $\left._{\mathrm{vB}}\right)$. Notons que le dérivé peut endosser deux rôles argumentaux distincts du procès exprimé, puisqu'il permet ici renvoyer au patient du procès, ou à un artefact servant à réaliser ce dernier.

\subsubsection{Bilan}

La sélection d'un participe passé en -et comme base de dérivation en breton contredit l'affirmation selon laquelle seuls des mots fléchis au pluriel pourraient accepter à leur tour une suffixation. Il convient d'observer que le formant -ed- peut représenter un suffixe nominal abstrait, un suffixe d'emprunt au français, une désinence infinitive ou encore plurielle, comme il a été vu. Ce formant paraît par conséquent particulièrement particulièrement prompt à accepter une suffixation en breton.

\subsection{La base sélectionnée est fléchie au féminin}

Une base portant la marque d'une flexion féminine peut également donner lieu à une double suffixation en -enn (suffixe du singulatif) ou en -ez (suffixe du féminin). Mentionnons maouez 'femme' > maouez-enn 'nana'29 (Favereau, 1992: 507b) et gwaz 'homme' > gwaz-enn 'gonzesse' (Favereau 1992: 303b). Le nom-base sur lequel ces dérivés sont construits désigne un individu de sexe féminin. L'apport du suffixe ne porte donc pas sur le genre du référent du dérivé. Ce dernier se laisse en réalité interpréter comme référent doté de propriétés lacunaires par rapport au référent du 
nom-base. Une nana n'est en effet pas encore une femme d'âge mûr, et ne possède pas les qualités (stabilité, expérience) de cette dernière. Le nom construit gwazenn suppose quant à lui une dérivation quelque peu différente, mais établie ici aussi sur une évalution péjorative du référent du nom-base. L'apport du suffixe sur le plan du genre est ici en effet actif, le nom-base étant de genre masculin ${ }^{30}$. Cet exemple fait écho au dérivé paotrezenn, recueilli par F. Favereau sous la plume de l'écrivain léonard YannVadezour Lagadeg ${ }^{31}$ :

(19) a. ur paotr > ur baotr-ez

un garçon une fille

b. ur baotr-ez > ur baotr-ez-enn

une fille une gamine de 10-12 ans

De même que dans gwazenn, où le suffixe permet de marquer le passage d'un référent masculin à un référent féminin, paotrez permet entre en effet en opposition flexionnelle avec paotr, sur le plan du genre (19-a) ${ }^{32}$. Cette instruction est en revanche neutralisée et redondante, lorsque le suffixe -enn opère sur paotrez (19-b). L'apport du suffixe semble alors être de nature diminutive - lacunaire ou appropriative ${ }^{33}-$. Un parallèle peut être fait également entre maouezenn et mammenn ou encore mammez:

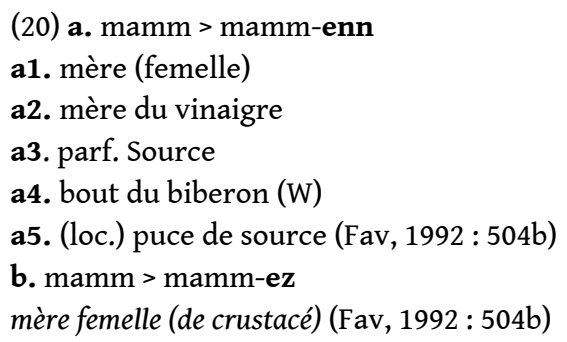

L'analyse de mamm-ez illustre le fait que les cas de neutralisation de l'instruction syntactique - flexionnelle - d'un suffixe, au profit d'une instruction particularisante ou évaluative - connotative - ne concernent pas seulement le suffixe -enn. Le suffixe $-e z$ est en effet traditionnellement décrit comme suffixe « habituel du féminin " (Favereau, 1997 : 78) permettant de créer des noms féminins sur la base de noms agentif en -er ou sur la base de noms non suffixés) :

(21) a. labourer > labourer-ez (Fav, 1997 : 78)

travailleur travailleuse

b. chef > chef-ez, -léon (relevés personnels)-

chef chef (féminin) ${ }^{34}$

Dans le dérivé mammez, la base de dérivation du dérivé est déjà, par elle-même, dotée du trait flexionnel [+fém.]. L'apport du suffixe ne peut, dès lors, porter sur le genre du dérivé. Trépos ([1956] $1982: 80$, n. 4) oppose ainsi mamm 'mère' à mammez 'femelle du crabe' ou mammez-voualc'h 'merlette'. Cet auteur fait remarquer que la flexion plurielle du nom mamm peut également permettre une différenciation sémantique :

Tableau 22

\begin{tabular}{|l|l|l|l|l|}
\hline & Nom singulier & Sémantisme & Flexion plurielle & Sémantisme \\
\hline a. & mamm & mère & mamm-où & mères \\
\hline b. & mamm-[lapined $]^{35}$ & mère lapine & mamm-ed [lapined] & mères lapines \\
\hline
\end{tabular}




\begin{tabular}{|l|l|l|l|l|}
\hline c. & mammez & femelle du crabe & mamm-izien (Douarnenez) & femelles du crabe \\
\hline d. & mammez-voualc'h & Merlette (Ploemeur) & [non renseigné] & [non renseigné] \\
\hline
\end{tabular}

Il y a lieu de penser que l'emploi du suffixe -ez permet de différencier une mère humaine d'une femelle animale. Le référent se laisse dès lors analyser comme type particulier du référent prototypique de la classe définie par le nom-base. Dans d'autres cas, ce redoublement peut induire une lecture évaluative :

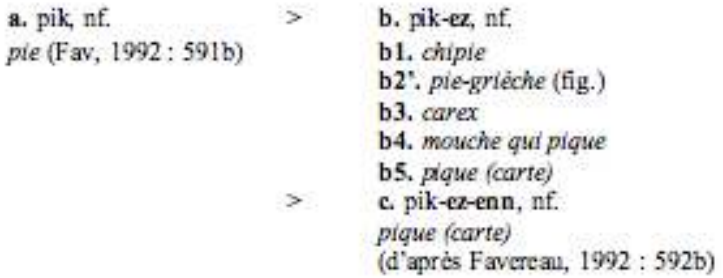

Ces dérivés ne se laissent pas tous analyser de la même façon. Les noms b1 et b2 peuvent être corrélés à pik 'pie', la pie étant caractérisée comme oiseau qui «jacasse bruyamment » (TLF). B4 renvoie en revanche à l'action de piquer exprimée par le verbe pikañ. B5 nous semble quant à lui être construit sur l'emprunt pique bretonnisé. Le dérivé $C$, enfin, pourrait s'expliquer par une volonté de différenciation entre pikez et pikezenn ${ }^{36}$.

Il ressort de ces données qu'un nom porteur du genre féminin peut accepter, en breton, de recevoir un suffixe lui-même porteur de cette information morphosyntaxique. La marque du genre féminin du nom support peut être exprimée par un suffixe ou par une mutation morphosyntaxique (le genre féminin de pik transparaît ainsi dans un syntagme déterminatif : ar 'la' ur 'une' + pik > ar bik ur bik). Il est remarquable noter le fait que l'apport sémantique de la suffixation peut ici être de nature référentielle ou évaluative, de la même manière que pour les doubles pluriels (cf. supra).

\subsection{Le dérivé conserve la marque d'une désinence verbale}

61 Un dérivé nominal ne peut être construit en breton sur une base porteuse d'une désinence infinitive:

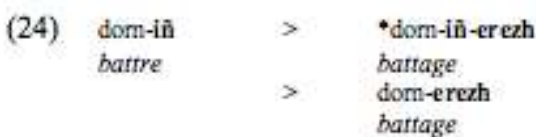

Dans un cas de figure particulier, néanmoins, le nom d'agent construit sur un verbe donné - de même parfois que le participe passé de ce dernier -, sont porteurs d'une marque - phonologique et/ou prosodique - permettant d'identifier la nature de la désinence du verbe-base.

L'analyse de la réalisation de pesketa (at) 'pêcher'37, pesketet pesketaet 'pêché', pesketaer pesketer pesketour 'pêcheur', dans divers points du Nouvel Atlas Linguistique de la Basse-Bretagne (op. cit.) permet d'aboutir au tableau suivant : 


\begin{tabular}{|c|c|c|c|c|c|}
\hline & \multicolumn{2}{|c|}{ Point d'enquête } & Pêcher & Pêché & Pêcheur \\
\hline a. & 11 & Bourg-Blanc (29) & [pes'kèta] & [pes'ketet] & [pes'kèt\&R] \\
\hline b. & 18 & Plounévez-Lochrist (29) & [pès'kèta] & - & [peske'ta:er] \\
\hline c. & 27 & Sibiril (29) & [pes'kètta] & [pès'kètət] & 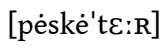 \\
\hline d. & 30 & Carantec (29) & [pès'kèta] & [pès'kètat] & [péskè'taعR] \\
\hline e. & 135 & Beuzec-Conq (29) & [pès'kètè] & [pès'kètət] & [pès'kètəR] \\
\hline f. & 152 & Ploërdut (56) & [pèskə'tæt] & [paska'tcjt] & [pèska'tu:R] \\
\hline
\end{tabular}

Il ressort tout d'abord que le participe passé du verbe-base ne porte aucune marque particulière, n'était au point 152 où il se réalise [zjt] et non [ət]. Il s'agit là d'une caractéristique commune à divers parlers du pays pourlet (56) notamment. Le participe passé non marqué est en effet régulièrement supplanté dans ces parlers ${ }^{38}$ par une forme diphtonguée lorsque le verbe-base est un verbe en -(h)at.

Si le participe passé ne porte aucune marque particulière dans les autres points d'enquête figurant dans ce tableau, il n'en va pas de même pour le nom agentif en -er dérivé de pesketa. Il ressort en effet que ce dernier peut être doté d'une accentuation oxytonique et non paroxytonique, comme cela est de rigueur dans ces parlers (cf. 23-b$c-d)$. La voyelle initiale $-a-$ de la désinence infinitive peut également être conservée dans le dérivé agentif et donner lieu à deux syllabes distinctes (23-b) ou à une diphtongue (23-d).

Le même phénomène peut se rencontrer lorsqu'un adjectif en -us est construit sur un verbe en -aat. Favereau (1992) mentionne par exemple abafaat 'complexer' > abafaüs (aux côtés d'abafus) (Favereau, 1992: 1b) ou encore ankounac'haat 'oublier' > ankounac'haüs 'oublieux' (Favereau, 1992 : 26a).

\subsection{Autres phénomènes de redoublement d'un marquage morphologique}

Les doubles pluriels, de même que les doubles singuliers, relèvent de processus mettant en jeu un double marquage morphologique. Les phénomènes de redoublement d'une marque morphologique sont relativement rares en breton.

Il est toutefois intéressant de noter le fait que ces derniers ne concernent pas exclusivement le domaine nominal. Trépos souligne ainsi le fait qu'un adjectif peut recevoir, dans certains cas, une double marque du comparatif. Le suffixe $-o c^{\prime} h \mathrm{du}$ comparatif régulier peut en effet parfois s'adjoindre à un comparatif irrégulier :

Tableau 26

\begin{tabular}{|l|l|l|l|l|}
\hline & Adjectif/adverbe & Comparatif régulier & Comparatif irrégulier & Double comparatif \\
\hline
\end{tabular}




\begin{tabular}{|c|c|c|c|c|}
\hline a. & $\begin{array}{l}\text { Brav } \\
\text { beau }\end{array}$ & $\begin{array}{l}\text { Brav-oc'h } \\
\text { plus beau }\end{array}$ & & \\
\hline b. & $\begin{array}{l}\text { Mad } \\
\text { bon }\end{array}$ & $\begin{array}{l}\text { Matoc'h } \\
\text { meilleur }\end{array}$ & $\begin{array}{l}\text { Gwell } \\
\text { meilleur }\end{array}$ & $\begin{array}{l}\text { Gwell-oc'h } \\
\text { meilleur }\end{array}$ \\
\hline c. & $\begin{array}{l}\text { Fall } \\
\text { mauvais }\end{array}$ & $\begin{array}{l}\text { Falloc'h } \\
\text { pire }\end{array}$ & $\begin{array}{l}\text { Gwazh } \\
\text { pire }\end{array}$ & $\begin{array}{l}\text { Gwash-oc'h } \\
\text { pire }\end{array}$ \\
\hline d. & - & - & $\begin{array}{l}\text { Mui } \\
\text { plus }\end{array}$ & $\begin{array}{l}\text { Mui-oc'h } \\
\text { plus }\end{array}$ \\
\hline
\end{tabular}

(d'après Trépos, $1982:$ 262)

Le redoublement d'une marque morphologique portant sur la personne exprimée n'est pas rare dans le système d'emploi des prépositions conjuguées. Fleuriot $(1989, \S 118)$ mentionne plusieurs prépositions conjuguées, porteuses d'une désinence personnelle explicite, et néanmoins suivies d'un pronom sujet :

Tableau 27

\begin{tabular}{|l|l|l|l|}
\hline & Préposition conjuguée & Pronom sujet \\
\hline a. & $\begin{array}{l}\text { Hepdo em } \\
\text { sans lui-lui }\end{array}$ & $\begin{array}{l}\text { Hepd-o } \\
\text { Sans lui }\end{array}$ & $\begin{array}{l}\text { Em } \\
\text { lui }\end{array}$ \\
\hline b. & $\begin{array}{l}\text { Dudo em } \\
\text { à lui-lui }\end{array}$ & $\begin{array}{l}\text { Dud-o } \\
\text { àlui }\end{array}$ & Em \\
\hline lui \\
\hline c. & $\begin{array}{l}\text { Conten ni } \\
\text { avec nous-nous }\end{array}$ & $\begin{array}{l}\text { Cont-en } \\
\text { avec nous }\end{array}$ & Ni \\
\hline
\end{tabular}

Ce type de redoublement est toujours très vivant en breton contemporain. La conjugaison des prépositions gant 'avec' et evit 'pour' est ainsi la suivante dans le parler de Scaër (29) ${ }^{39}$

Tableau 28

\begin{tabular}{|l|l|l|l|l|}
\hline \multicolumn{2}{|l|}{ Pronom gant 'avec' } & \multicolumn{2}{l|}{ Pronom evit 'pour' } \\
\hline & $\begin{array}{l}\text { Forme } \\
\text { emphatique }\end{array}$ & $\begin{array}{l}\text { Forme } \\
\text { emphatique } \\
\text { emphatique }\end{array}$ & $\begin{array}{l}\text { Forme } \\
\text { emphatique }\end{array}$ \\
\hline 1 & Ganeñ & $\begin{array}{l}\text { Ganin-me } \\
\text { Ganin-men }\end{array}$ & Eviteñ & $\begin{array}{l}\text { Evitin-me } \\
\text { Evitin-men }\end{array}$ \\
\hline 2 & - & - & - & - \\
\hline
\end{tabular}




\begin{tabular}{|l|l|l|l|l|}
\hline 3 & Gaton & - & Evitoñ & - \\
\hline 3 fém & Gati & - & Eviti & - \\
\hline 1 & Ganomp & Ganomp-ni & Evidomp & Evidomp-ni \\
\hline 2 & Ganoc'h & Ganoc'h-c'hwi & Evidoc'h & Evidoc'h-c'hwi \\
\hline 3 & Gate & - & Evite & - \\
\hline
\end{tabular}

Conjugaison des prépositions gant et evit à Scaër (29) 40

\section{Conclusion}

Il est temps pour nous de conclure cet article en reprenant les différents points qui ont été défendus :

1. La morphologie bretonne illustre le fait qu'une unité porteuse d'une flexion plurielle peut être sélectionnée comme base de dérivation. Cette sélection ne peut se faire toutefois que sous certaines conditions.

2. La sélection d'une base porteuse d'un trait flexionnel morphologique donné, par un suffixe porteur du même trait n'est pas propre aux noms pluriels. La dérivation de faux-singulatifs en -enn construits sur un nom féminin illustre, notamment, cette possibilité

3. L'apport du double pluriel ou du double féminin peut être de nature référentielle, mais également évaluative voire énonciative (cf. Pôle Interlocuteur)

4. Les participes passés en -et peuvent donner lieu en breton à divers types de suffixations

5. Une partie des données étudiées dans cet article relève de la problématique du redoublement d'une marque morphologique ou grammaticale. Ces redoublements ne concernent pas le domaine nominal exclusivement puisqu'ils peuvent également concerner des adjectifs ou des prépositions fléchies.

\section{BIBLIOGRAPHIE}

\section{Linguistique}

Acquaviva, P. 2005. « The morphosemantics of transnumeral nouns ». Booij, G., Guevara, E., Ralli, A., Sgroi, S. and Scalise, S (eds.). Morphology and Linguistic Typology. On-line Proceedings of the Fourth Mediterranean Morphology Meeting (MMM4) University of Bologna, pp. 252-265.

Acquaviva Paulo, 2008. Lexical Plurals, A Morphosemantic Approach. Oxford Studies in Theoretical Linguistics. 
Anderson, Stephen R. 1982. « Where’s Morphology ? ». Linguistic Inquiry 13, pp. 571-612.

Anderson, Stephen R. 1986. « Disjunctive Ordering in Inflectional Morphology ». Natural Language and Linguistic Theory 4, 1-31.

Belder, Marieke De, 2010. « On Breton pluralization ». Manuscrit non publié.

PDF consulté la dernière fois le 7/03/11 :

http://www.marijkedebelder.com/marijkedebelder.com_downloads.html

Bochner, Harry, 1984. «Inflection within Derivation ». The Linguistic Review 3, pp. 411-421.

Di Sciullo, Anna Maria, et Edwin Williams, 1987. On the Definition of Word. MIT Press, Cambridge, Massachusetts.

Fradin Bernard et Kerleroux Françoise, 2009. «L'identité lexémique ». Aperçus de Morphologie du français, sous la direction de Bernard Fradin, Françoise Kerleroux et Marc Plénat, pp. 83-103.

Fradin Bernard, 2003a. Nouvelles approches en morphologie, Linguistique nouvelle, PUF

Fradin Bernard, 2003b. « Le traitement de la suffixation en -ET ».

(PDF disponible en ligne : http://www.llf.cnrs.fr/Gens/Fradin/index-fr.php ). Paru dans Langages, 2003.

Greenberg, Joseph H. 1963. « Some universals of grammar with particular reference to the order of meaningful elements. In Universals of Language, ed. par Joseph H. Greenberg, 73-113. Londres : MIT Press.

Matthews, P. H., 1972. Inflectional Morphology. Cambridge University Press, Cambridge.

Morris Jones John, [1913] 1955. A Welsh Grammar. Historical and Comparative. Oxford, 1913, repr. 1955.

Perlmutter, David. 1988. «The Split Morphology Hypothesis : Evidence from Yiddish ». Michael Hammond et Michael Noonan, éds. Theoretical Morphology : Approaches in Modern Linguistics. Academic Press, San Diego.

Stump Gregory T., 1984. « Agreement vs. Incorporation in Breton ». Natural Language and Linguistic Theory 2, pp. 289-348.

Stump Gregory T., 1989. « A note on Breton pluralization and the elsewhere condition ». Natural Language \& Linguistic Theory, Springer Netherlands, Volume 7, Number 2.

Stump Gregory T., 1990. « La morphologie bretonne et la frontière entre la flexion et la dérivation ». La Bretagne Linguistique 6, 185-237.

Stump Gregory T, 1993. « How Peculiar is Evaluative Morphology? ». Journal of Linguistics 29(1). Wiltschko Martina \& Steriopolo Olga, 2007. «Parameters of variation in the syntax of diminutives ». Proceedings of the 2007 annual conference of the Canadian Linguistic Association.

\section{Domaine breton}

Deshayes Albert, 2003. Dictionnaire étymologique du breton. Ed. Chasse-marée, Douarnenez.

Ernault Emile, 1897. Petite Grammaire Bretonne, Imprimerie René Prud'homme, Saint-Brieuc.

Ernault Emile, [1909], 3ème éd. 1991. Dictionnaire Breton-Français du dialecte de Vannes, Brud Nevez, Brest. 
Favereau Francis, 1992. Dictionnaire du Breton Contemporain, Skol Vreizh, Morlaix.

Favereau Francis, 1997. Grammaire du breton contemporain. Ed. Skol Vreizh, Morlaix.

Fleuriot Léon, [1964] 1989. Le vieux breton. Eléments d'une grammaire. Slatkine Reprints. GenèveParis, 439p.

Le Roux Pierre, 1924-1963. Atlas Linguistique de la Basse-Bretagne, Plihon-Hommay, Rennes, Paris (consultable en ligne à l'adresse suivante : http://sbahuaud.free.fr/ALBB/)

Ménard Martial et Cadoret Iwan (sous la direction de), 2001. Geriadur brezhoneg. Editions an Here, Plougastel-Daoulas.

Press Iann, 1947. A Grammar of Modern Breton, Mouton de Gruyer.

Riou Yann, 2004. Echos du bord de mer, Mémoire, culture et langue bretonne à Lampaul-Plouarzel, Emgleo Breiz, Brest.

Trépos Pierre, [1956], 1982. Le Pluriel Breton, Ed. Brud Nevez, Brest

Trépos Pierre, [1968], 1996. Grammaire bretonne, $5^{\text {ème }}$ édition, 1996. Ed. Brud Nevez, Brest

Wmffre, Y., 1998. Central Breton. Munich : Lincom Europa.

\title{
ANNEXES
}

\section{Symboles et abréviations}

\author{
ALBB Atlas Linguistique de la Basse-Bretagne (op. cit.) \\ DIM Diminutif \\ PL Pluriel
}

SG Singulier

\section{NOTES}

1. «Du point de vue de la dimension morphosyntaxique, l'entité linguistique qui sert de base aux RCL [NB. Règles de Construction de Lexèmes] est une unité qui n'est pas spécifiée pour la flexion, puisqu'elle est située hors emploi. Cette propriété se manifeste par le fait que les marques flexionnelles, en tant qu'elles sont l'exposant de propriétés morphosyntaxiques, ne sont jamais à prendre en compte dans la caractérisation de la forme du lexème (Matthews 1974). Elles ne sont pas pertinentes en Morphologie constructionnelle. » (Fradin \& Kerleroux, 2008 : 84)

2. Cité par Stump $(1990: 195)$ et traduit en français par ce dernier : «(La théorie proposée) représente le fait, souvent noté mais jamais vraiment expliqué, que (à part quelques exceptions bien définies) la morphologie flexionnelle parait "en dehors de" la morphologie dérivationnelle. Par exemple, si une structure morphologique complexe contient et des suffixes dérivationnels et des suffixes flexionnels, ceux-ci suivront ceux-là [...] Ce n'est que lorsque dans le cas des procédés flexionnels qui spécifient des changements à l'intérieur des thèmes (tels que l'apophonie, la métaphonie, ou les alternances parmi les configurations verbales caractéristiques des langues sémitiques) ou insèrent de véritables infixes qu'il est possible qu'un élément formatif flexionnel 
paraisse "à l'intérieur" d'une formation dérivationnelle. ». Nous ne sommes pas en mesure de déterminer avec précision à quels phénomènes Anderson fait ici référence.

3. Cf. Stump, passim pour des tests explicites)

4. La voyelle initiale du suffixe -at peut également parfois être conservée dans le déverbal agentif, d'où la possibilité d'obtenir une dérivation telle que : merc'heta $>$ merc'heta $>$ merc'heta-er (cf. infra pour une discussion).

5. Cf. Stump (1998: 18-22) par exemple sur ce point.

6. Acquaviva ne nous semble pas analyser, de manière explicite, les suffixes pluriels insérés à l'intérieur de mots construits comme suffixes dérivationnels. L'interprétation défendue par cet auteur est plutôt que les suffixes pluriels sont bien des marqueurs flexionnels, mais d'un type spécifique : «[...] the plural that appears in the body of a word is a special type of inflectional marker - one used as a lexical formant. » (Acquaviva, $2008: 243$ ).

7. Les cardinaux ont la particularité, en breton, d'imposer que le nom qu'ils déterminent soit de nombre singulier.

8. Nous mettons en doute le fait que le pluriel simple et le double pluriel possèdent également la même signification - ou la même distribution syntaxique - aux yeux d'Ernault, les autres pluriels bugaleoù et paotredoù ayant, quant à eux, un sens spécifique, chez cet auteur. Aussi attribuonsnous cette traduction à une négligence de l'auteur.

9. Nous relevons nous-même l'emploi de merc'hedoù avec un sens plutôt péjoratif en pays pourlet (Inguiniel, 56) : tammoù merc'hed digampenn. A, lâret merc'hedoù zod ar re-mañ !, -Inguiniel, 56- // espèces de+filles+au comportement incorrect. Ah, quelles+n-ed-où+sottes+celles-ci // Des filles peu estimables, au comportement incorrect. Ah, tu parles, quelles gourdasses ces filles-là !

10. Le gallois possède un suffixe -os permettant de créer des noms collectifs. Ce suffixe, généralement adjoint à des noms pluriels, permet, d'après Morris-John (1921), de créer des doubles pluriels exprimant une notion de mépris ou d'affection. Le tableau situé en Annexes (Tableau note 10 à téléharger) illustre le parallèle frappant qu'il est possible d'effectuer entre les doubles pluriels bretons et gallois (d'après Zimmer (2000: § 64.5)

11. Attesté également dans l'emploi du double pluriel tudoù 'gens+-où' dans divers dialectes.

12. Si le singulier roñse est bien attesté dans certains parlers (Favereau, 1992: 644b), il a été supplanté dans d'autres parlers par l'emploi d'un autre terme (jao 'cheval (de sexe indifférencié) vs roñsed 'chevaux' par exemple).

13. La non discernabilité des unités comprises dans une pluralité - et l'impression de confusion qui en ressort - est donc, pour Trépos, une caractéristique définitoire des noms collectifs en breton.

14. La sélection de bugale comme $\mathrm{PL}_{1}$ susceptible d'être "repluralisé ", - pour reprendre le vocabulaire d'Acquaviva 2008 - en raison d'une perception de ce nom comme collectif peut s'expliquer par divers arguments. Dans certains dialectes tout d'abord, le nom singulier bugel n'est plus employé librement (dialecte pourlet d'Inguiniel 56 par exemple). Il ressort par ailleurs que la base bugale- donne lieu à diverses dérivations: bugale-at 'enfanter', bugale-adur 'enfantement', bugale-aj 'enfance', bugale-erezh 'enfance', là on l'on attendrait - sur des critères sémantiques, et au vu du nombre des bases normalement sélectionnées par ces suffixes respectifs - la sélection du nom singulier bugel- (Favereau, 1992 : 96a). La sélection de ce radical infléchi est donc idiosyncrasique. Sur un plan sémantique, enfin, bugale renvoie à une totalité indéterminée et non discrète sur un plan interne.

15. Qui a évolué en -eier dans certains dialectes.

16. Le diacritique que nous utilisons ici indique que la base bot- n'est pas autonome, sur un plan morphosyntaxique. Cette dernière se laisse interpréter, toutefois, comme transparente, sur un plan diglossique (dans une situation de bilinguisme breton/français) supposée. Nous pensons qu'un grand nombre de bases non autonomes ont en réalité le statut de bases transparentes en breton. 
17. Une étude approfondie - étayée par des compléments d'enquête - serait nécessaire pour déterminer avec précision le caractère sémantique (et syntaxique) de l'opposition entre $\mathrm{N}$-où et $\mathrm{N}$-oùier dans les différents dialectes mentionnés ici.

18. Il est à noter que Trépos (1982) mentionne un autre type d'opposition entre pluriels discontinus du singulatif en -ennoù et pluriels indéterminés (ou collectifs) en -ier (accompagnés d'une inflexion vocalique). Il conviendrait de déterminer dans quelle mesure l'opposition entre ces deux types de pluriels est productive dans des dialectes déterminés (voir Annexes: Tableau note 18 à télécharger). D'autres oppositions entre un N-où et un N-er sont possibles, sans qu'une double pluralisation soit nécessaire. Un informateur de Scaër (29) que nous avons consulté oppose ainsi '[gwern]-ioù 'des marais [+ nombre déterminé ; paucal]' à '[gwern]-ijer 'des marais [+ grand nombre; nombre indéterminé]'.

19. Notons que Trépos souligne le fait, après Pedersen, que l'on trouve trace de tels pluriels en cornique également. Cf. flehesygow, « des petits pages » (Vergleichende Grammatik der Keltischen Sprachen, von Holger Pedersen, Göttingen, 1909, 1913, § 412, n. 3). Trépos précise que l'on trouve mention de doubles pluriels du diminutif tout autant que de diminutifs pluriels simple en moyen breton. Ces derniers seraient néanmoins nettement majoritaires en toponymie (l'exception relevée par Trépos étant Poullouigou « Petites Mares », formé sur poullig).

20. «c'est que ces pluriels répondent à un besoin précis : une première marque du pluriel, entre le singulier et le suffixe diminutif, évite la création de l'image du singulier ; ni l'article en effet, ni le possessif, lorsqu'il y en a, ne donne de précision sur le nombre, et, dans le premier type, la marque du pluriel arrive bien tard, après l'image du singulier, puis du diminutif (prad-ig-ou, « un pré - petit - plusieurs »); dans certains cas, cette image du singulier était gênante, et le Breton a préféré évoquer immédiatement l'image du pluriel ; ces formes prad-ou-igou pourraient s'analyser «des prés - petits - plusieurs », et rappellent les pluriels formés sur les singulatifs : stered-enn-ou, «des étoiles - une à une - plusieurs » : pluriel + suffixe singulatif ou suff. diminutif + suff. pluriel. » (Trépos, 1982 : 275)

21. Les catégories du diminutif et du singulatif partagent de nombreux points communs en breton et en gallois. Une généalogie commune - ou tout au moins imbriquée - sur un plan étymologique, tout autant que sémantique, peut être proposée pour ces deux catégories (cf. Cuzzolin, 1998 ; Irslinger 2010).

22. Cette pastille indique que le dérivé est perçu comme possible par le locuteur interrogé :

- moaiand zo lâret yerigoù bihan, me' 'meus ket bet klavet pikol, -Berné, 56-

On peut dire "des petites poules", mais je ne l'ai pas beaucoup entendu

Notons que le N-igoù est suivi ici de l'adjectif bihan. La marque du diminutif est ici redoublée, par conséquent.

23. Un relecteur nous affirme avoir relevé la forme zaoudigoù dans une commune du Bas Vannetais.

24. Les formes maouzedigoù et evnedigoù sont parfaitement acceptées par un autre informateur interrogé en pays pourlet (Inguiniel, 56).

25. Stump (1990) note que la forme chasigoù - var. de chachigoù dans certains dialectes - a été refusée par des informateurs interrogés à Plougastel.

26. «[...] the simple plural always precedes derivational suffixes (Stump 1989 : 272). This is exactly what we expect ; a stem allomorph cannot be broken apart by derivation. » (Belder, $2010: 8$ ).

27. Zimmer (2000: 488) montre que l'emploi du suffixe - ed - dérivé du suffixe proto-indoeuropéen -(a)tó- a été inclus, en gallois, dans le paradigme verbal des formes impersonnelles du prétérit (ex. ganed 'was born'). Les emplois du suffixe -ed comme suffixe du participe passé sont très rares en gallois - le suffixe -edig ayant acquis cette fonction - contrairement à ce que l'on observe en breton où -ed est resté la forme du participe passé.

28. La terminaison -aith attestée dans ce suffixe est issue de *aktā « acte, action » d'après Fleuriot (1989 : 338). 
29. Ce dérivé est étymologiquement un «triple singulier » puisqu'il est construit sur l'adjectif et nom maw 'gaillard', issu du vieux breton mauu 'juvénile, réjoui ; jeune homme' (Deshayes, 2003 : 498a).

30. Notons au passage que le dérivé gonzesse est lui-même formé sur le nom masculin gonze désignant, en argot, un «homme en général, un individu » (TLF en ligne, cf. lexilogos.fr). Sur le plan référentiel, ce N-esse peut renvoyer à une «Femme en général ; fille, parfois de mœurs légères ; maîtresse » mais également à un « homme lâche, couard » (TLF, id.).

31. Communication personnelle du 10-10-09.

32. L'aire d'emploi du dérivé paotrez pour référer à une fille au sens de jeune personne de sexe féminin (et non à une fille sur le plan de la filiation), est documentée par la carte 447 de l'ALBB.

33. Seuls des exemples explicites, sur un plan discursif, permettraient de trancher.

34. Trépos ([1956] 1982: 85 sqq.) mentionne également le cas des noms féminins nus faisant leur pluriel en -ez-ed (e.g. c'hoar 'sœur' > c'hoar-ez-ed 'sœurs') voire de noms en féminins en -ez dérivés, étymologiquement, d'un nom singulier nu (e.g. m. br. mazron 'marraine' > bret. mod. maeron-ez 'id.', pl. maeron-ez-ed) (Trépos, id. : 262). Trépos évoque le fait que le suffixe -ez- de c'hoarezed par exemple a pu donner lieu à deux interprétations. Pour Falc'hun par exemple, il s'agirait d'un représentant du suffixe pluriel -ez correspondant au gallois -yedd, -edd ou -oedd (e.g. gallois chwaer 'sœur', pl. chwiorydd). Cette hypothèse est rejetée par Trépos qui privilégie l'interprétation de ce suffixe comme représentant du suffixe féminin -ez. Pour le grammairien, l'ajout de la séquence -ez-ed à des noms singuliers dénués du suffixe -ez serait de nature analogique : «Il se trouve [...] que la plupart des noms féminins d'êtres animés se terminent au pluriel par -ez-ed: lorsque le singulier n'est pas en -ez, le pluriel en -ed parait incomplet au bretonnant, qui utilise le suffixe composé -ezed, auquel il est accoutumé » (Trépos, [1956] 1982 : 85). Cette hypothèse serait également celle de Pedersen (Pluralendung + überflüssiger Femeninendung (VGKS, II, p. 71, n . 3) (id. 86).

35. Nous ajoutons [lapined] à la citation de Trépos qui traduit mammed par 'mères lapines', ce qui laisse supposer que la mention du N2 classifiant n'est pas obligatoire.

36. Nous ne relevons que de rares dérivés artefactuels en -ezenn chez Favereau (1992). Mentionnons kostez 'côté' > kostez-enn 'côte (\& côtelette), côté (\& parti), parf. filière' (Fav, 1992 : 434a).

37. Le verbe pesketa - var. pesketa-at - est porteur de la désinence -(h)a héritée d'un " ancien verbe ham 'appréhender' » (Favereau, $1997: 174)$.

38. Observations personnelles à Berné (56) et Inguiniel (56).

39. D'après l'idiolecte de l'informateur TA.

40. D'après des observations personnelles.

\section{RÉSUMÉS}

Il est établi de longue date, dans le domaine de la morphologie, que la dérivation précède la flexion, et non l'inverse (Greenberg, 1963 : 83). L'on doit à Stump $(1989 ; 1990)$ notamment d'avoir souligné le fait que les données bretonnes pouvaient échapper, pour partie, à cet universel. Cette interprétation a fait l'objet d'une réévaluation récente de la part d'Acquaviva (2008) pour lequel seules des unités relevant de la flexion du nombre peuvent, en breton, être mobilisées comme bases dérivationnelles. Pour Belder (2010), la flexion ne précéderait pas la dérivation en breton, 
le pluriel $_{1}$ précédant un pluriel ${ }_{2}$ n'ayant d'autre statut, dans cette langue, que celui d'un allomorphe thématique. L'objectif qui est le nôtre dans cet article est de démontrer que la sélection d'une base fléchie n'est pas propre, en breton, aux noms pluriels. Elle s'intègre, dans certains cas, à une problématique plus générale de redoublement d'une même marque morphologique.

In the field of morphology, it has been known for a long time that derivation precedes inflexion, and not the other way round (Greenberg 1963: 83). Among others, Stump (1989, 1990) emphasized the fact that parts of the Breton language data may be exceptions to that rule. This opinion was discussed again, quite recently, by Acquaviva (2008), who thinks that only the number inflexion units may be used as derivation roots in Breton. In Belder's view (2010), inflexion may not precede derivation in Breton, since the plural 1 that precedes a plural 2 has no status in the language except that of a thematic allomorph. In this paper, my aim is to demonstrate that the selection of an inflected root does not only apply to plural nouns, in Breton. In certain cases, it falls within the more general problematics of reduplication of one single morphological mark.

INDEX

Keywords : morphology, derivation, inflexion, lexeme

Mots-clés : morphologie, dérivation, flexion, lexème

\section{AUTEUR}

PIERRE-YVES KERSULEC

Université de Rennes 2, CRBC FRE 3055 